J. Klin. Endokrinol. Stoffw. 2020 - 13:77-81

https://doi.org/10.1007/s41969-020-00101-0

(c) Der/die Autor(en) 2020

\author{
Alicia Baumgartner' - Sabina Baumgartner-Parzer ${ }^{2}$ \\ 'Wien, Österreich \\ ${ }^{2}$ Universitätsklinik für Innere Medizin III, Medizinische Universität Wien, Wien, Österreich
}

\title{
GNAS-assoziierte Erkrankungen
}

\section{GNAS-Komplex}

Der auf Chromosom 20q13.2-13.3 gelegene GNAS-Genlokus (Guanine nucleotide-binding protein, alpha-stimulating activity polypeptide 1) zeigt einen äußerst komplexen Aufbau, mit unterschiedlichen sich überlappenden Transkripten (eines davon "antisense“ - d.h. vom komplementären DNA-Strang) und mehreren Promotoren. Zudem stellt der GNAS-Komplex einen Imprinting-Lokus dar, der zwar primär biallele, aber ebenso rein maternale oder paternale Transkripte exprimieren kann. Das bekannteste Genprodukt ist das Gsa, das eine Untereinheit des Guanosintriphosphat-bindenden Proteins (G-Protein) darstellt und die Adenylatcyclase stimuliert. Letztere ist maßgeblich an der Regulierung der Aktivitäten endokriner Drüsen (z. B. Schilddrüse, Hypophyse, Gonaden, Nebenniere, etc.) wie auch an der Knochenentwicklung beteiligt, da ihr eine wichtige Rolle in einer Reihe von Signaltransuktionswegen transmembraner Rezeptoren zugeschrieben wird [1-3].

Ebenso komplex wie der Genlokus ist auch das Mutationsspektrum von GNAS, das zu einer Reihe unterschiedlicher Erkrankungen führt (siehe - Tab. 1).

\section{GNAS-Mutationen und Krankheitsspektrum}

Die Gsa-Untereinheit des G-Proteins, welche ubiquitär exprimiert wird, ist durch seine Rolle in Signaltransduktionswegen maßgeblich an der Wirkung vieler Hormone und endogener Moleküle beteiligt. Pathogene Varianten (Mutationen), die die Expression bzw. Aktivität von Gs $\alpha$ beeinträchtigen, führen zu seltenen angeborenen Erkrankungen, wobei unter dem Überbegriff Albright'sche hereditäre Osteodystrophie (AHO) eine Reihe verschiedener Subtypen von Pseudohypoparathyreoidismus (PHP) wie auch Pseudopseudohypoparathyreoidismus zusammengefasst werden.

Prinzipiell kann betreffend pathogener Varianten wie folgt unterschieden werden:

Eine Keimbahnmutation tritt erstmals als Fehler bei Reifeteilung und DNA-Replikation in den weiblichen oder männlichen Keimzellen auf. Sie wird bei der Befruchtung an die Zygote und in Folge bei sämtlichen Zellteilungen an alle Zellen weitergegeben. Daher sind Keimbahnmutationen in allen Körperzellen konstitutiv nachweisbar und auch vererbbar. Sind diese Varianten mit einer Funktionseinschränkung verbunden, werden sie als pathogene Varianten einer erblichen Erkrankung eingestuft, die prä- und postnatal detektiert werden können.

Somatische Mutationen entstehen in teilungsaktiven Zellen der unterschiedlichsten Gewebe und sind auf diese beschränkt, sodass sie auch nur in den betroffenen Zellen im entsprechenden Gewebe nachweisbar sind. Häufig sind sie die Ursache für (sogenannte sporadische) Tumoren in bestimmten Geweben (bestimmter Organe) im Gegensatz zu erblichen Tumorsyndromen, bei denen durch das Vorliegen einer Keimbahnmutation auf einem Allel eine Prädisposition für einen Tumor besteht (Two-hit-Hypothese - vergleiche MEN1).

Postzygotische Mutationen treten erstmals in der Zygote oder in den ersten Teilungsstadien auf und werden in der Folge lediglich auf bestimmte Keimblätter verteilt, wodurch ein sogenanntes genetisches Mosaik entsteht [4]. Dies bedeutet, dass, obwohl die Zellen von der identen befruchteten Eizelle stammen, bestimmte Genotypen bzw. Genvarianten lediglich in bestimmten Zellen (Geweben) und nicht in allen Körperzellen vorliegen und nachgewiesen werden können.

Das mutationsbedingte Krankheitsspektrum von GNAS ist aus - Tab. 1 abzulesen, wobei GNAS-Keimbahnmutationen $\mathrm{zu}$ verschiedenen Formen des Pseudohypoparathyreoidismus führen, während somatische Mutationen für das McCune-Albright-Syndrom verantwortlich gemacht werden.

Aufgrund der Komplexität des oben beschriebenen Genlokus wurden erst in letzter Zeit signifikante Fortschritte betreffend der klinischen Auswirkungen erzielt. Dadurch wurde eine bessere Differenzialdiagnose betreffend andere fibroossäre Skelettläsionen oft erst möglich. Rezente Forschungsergebnisse legen die Vermutung nahe, dass pathogene Varianten des GNAS-Komplexes auch noch bei weiteren ähnlichen Erkrankungen eine Rolle spielen könnten.

Unter dem Überbegriff des Pseudohypoparathyreoidismus mit Albright'scher hereditärer Osteodystrophie (AHO) finden sich PHP-1-Subtypen wie auch andere unten aufgelistete seltene Krankheitsbilder.

\section{Pathophysiologie und klinische Symptomatik}

Pseudohypoparathyreoidismus Typ1a und PHP-1c sind durch eine Hormonresistenz (Parathormon, aber auch andere Hormone und Neurotransmitter) gekennzeichnet, ebenso durch eine AHO und eine Brachydaktylie Typ E. Die Subtypen 1a und 1c zeigen einen sehr ähnlichen Phänotyp (siehe • Tab. 2), unterscheiden sich aber betreffend ihrer Gsa-Aktivität, die bei PHP-1a vermin- 


\begin{tabular}{|c|c|c|c|}
\hline Erkrankung & GNAS-Mutation & ORPHA & OMIM \\
\hline Pseudohypoparathyreoidismus la (PHP-la) & Keimbahn & 79443 & 103580 \\
\hline Pseudohypoparathyreoidismus Ib (PHP-lb) & Keimbahn & 94089 & 603233 \\
\hline Pseudohypoparathyreoidismus Ic (PHP-Ic) & Keimbahn & 79444 & 612462 \\
\hline $\begin{array}{l}\text { Primärer Pseudopseudohypoparathyreoidismus (PP- } \\
\text { HP) }\end{array}$ & Keimbahn & 79445 & 612463 \\
\hline Progressive ossäre Heteroplasie (POH) & Keimbahn & 2762 & 166350 \\
\hline $\begin{array}{l}\text { Cushing-Syndrom durch makronoduläre Nebennieren- } \\
\text { hyperplasie }\end{array}$ & Somatisch & 189427 & 219080 \\
\hline Knochendysplasie, fibröse polyostotische & Somatisch & 93276 & - \\
\hline McCune-Albright-Syndrom & Somatisch & 562 & 174800 \\
\hline Mazabraud-Syndrom & Kandidatengen & 57782 & - \\
\hline
\end{tabular}

dert ist. PHP-1a/c, PPHP und $\mathbf{P O H}$ werden durch inaktivierende Loss-offunction-Mutationen (pathogene Varianten) hervorgerufen [5], worauf später noch näher eingegangen wird.

Da Ossifikationsstörungen $(\mathrm{POH})$ jedoch mit der Vererbung durch das paternale Allel assoziiert sind, wird abgeleitet, dass progressive Osteoblastendifferenzierung und die Proliferation der Zellen im Bindegewebe spezifisch vom väterlichen Allel beeinflusst werden [6, 7].

PHP-1b wird maternal vererbt (siehe - Tab. 2) und ist durch eine PTH-Resistenz charakterisiert, bei manchen PatientInnen liegt aber auch eine partielle Thyreoidea stimulierendes Hormon(TSH)Resistenz und eine Brachydaktylie vor.

Osteoma cutis ist eine seltene Erkrankung mit auf Knochenbildung in der Haut und Subkutis beruhenden Tumoren.

POH überschneidet sich mit einer Reihe verwandter genetischer Störungen einschließlich Albright'scher hereditärer Osteodystrophie, Pseudohypoparathyreoidismus und primären kutanen Osteomen, die ebenfalls mit einer oberflächlichen heterotopen Ossifikation und inaktivierenden Varianten des GNASGens einhergehen. Klinisch kann die $\mathrm{POH}$ jedoch abgegrenzt werden, da sie im fortgeschrittenen Stadium auch subkutanes und tiefes Bindegewebe einschließlich Muskeln und Faszien betrifft.

Das McCune-Albright Syndrom (MAS) wird durch aktivierende GNASVarianten (postzygotischer bzw. somatischer Natur) verursacht, die in einer verminderten intrinsischen GTPase-
Aktivität münden und so zu einer Überstimulation der entsprechenden Gewebe führen.

So kommt es zu erhöhter osteogener Bindung der Stromazellen, verminderter Differenzierung in Osteoblasten, wodurch fibröse dysplatische Läsionen entstehen, die frühe osteoblastische Marker (z.B. alkalische Phosphatase) exprimieren. Ebenso treten die Hautläsionen als Ergebnis einer erhöhten Gsa-Signalübertragung auf, die normalerweise über das melanozytenstimulierende Hormon die Melaninproduktion vermittelt.

Klinisch zeichnet sich das MAS durch die klassische Trias der polyostotischen fibrösen Dysplasie (FD), der Café-au-laitHautpigmentierung und der peripheren vorzeitigen Pubertät aus. Zusätzlich können Hyperthyreose, Cushing-Syndrom und Hypophysen-Gigantismus/ Akromegalie Teil der klinischen Präsentation sein, wenn mutierte Zellen in Schilddrüsen-, Nebennieren- und/oder Hypophysengewebe vorhanden sind.

Die meist zwischen dem 5. und 15. Lebensjahr beginnende Ossifikationsstörung betrifft vor allem die langen Röhrenknochen (Auftreibungen, Deformierungen) und den Schädel. Neben Kleinwuchs, kurzem Hals, rundem Gesicht und gedrungenem Körperbau mit Adipositas zeigen sich die charakteristischen großflächigen Café-au-laitFlecken (im Gegensatz zur Neurofibromatose Typ 1 [NF1] landkartenförmig unregelmäßig begrenzt) oft an Gesäß, Oberschenkeln und Rücken wie auch subkutane Verkalkungen. Die Hautläsionen zeigen typischerweise eine asymme- trische Verteilung entlang der BlaschkoLinien und überschreiten nicht die Mittellinie.

Ebenso werden Zeichen der chronischen Hypokalzämie (Tetanien), intrakranielle Verkalkungen, Zahnschmelzanomalien, Brachydaktylie oder auch eine psychomotorische Retardierung beobachtet.

Das Mazabraud-Syndrom ist charakterisiert durch das vergesellschaftete Auftreten der fibrösen Knochendysplasie (poly-, aber auch monoostotisch) mit intramuskulären Myxomen (Tumoren aus embryonalem Schleimgewebe) im jüngeren Lebensalter während der Wachstumsphasen und kann sich sowohl asymptomatisch als auch mit Schmerzen, Frakturen oder Skelettdeformitäten präsentieren. Im Erwachsenenalter imponieren multifokale intramuskuläre Myxome als schmerzlose Weichteilmassen - am häufigsten in den unteren Extremitäten.

\section{Genetische Diagnostik}

\section{GNAS-Allel-spezifische Vererbung - Imprinting}

Das auf Chromosom 20q13.32 gelegene Gen GNAS complex locus (OMIM 139320) ist ein Imprinting-Lokus. Imprinting ist ein epigenetisches Phänomen, das dazu führt, dass bei bestimmten Genen das Allel eines Elternteils durch eine spezifische Methylierung inaktiviert ist und das Gen daher von diesem Allel nicht exprimiert wird. Der GNASKomplex umfasst die Haupttranskripte $G s \alpha, X L A S, N E S P 55$ und $A / B$, von denen lediglich Gsa nicht imprintet ist.

- Gsa, das bekanntestes Genprodukt, wird biallellisch exprimiert und fungiert als Modulator verschiedener Signaltransduktionsprozesse.

- XLAS (large G-protein XL- $\alpha$ ) ist eine große Variante von Gsa, das ausschließlich vom paternalen GNASAllel primär in neuroendokrinem Gewebe und im Nervensystem exprimiert wird.

- NESP55 (Neuroendokrines sekretorisches Protein-55) wird exklusiv vom maternalen Allel exprimiert und kodiert ein Chromogranin- 


\begin{tabular}{|c|c|c|c|c|c|}
\hline \multirow[t]{2}{*}{ Klinik } & \multicolumn{3}{|l|}{ Charakteristika } & \multirow{2}{*}{$\begin{array}{l}\text { Expression } \\
\text { M(aternal) } \\
\text { P(aternal) }\end{array}$} & \multirow[t]{2}{*}{ Gendefekt } \\
\hline & Endokrine & Klinisch & Andere & & \\
\hline PHP-la & $\begin{array}{l}\text { Multihormon- } \\
\text { resistenz }\end{array}$ & $\begin{array}{l}\text { AHO } \\
\text { Adipositas }\end{array}$ & $\begin{array}{l}\text { Kognitive Einschränkun- } \\
\text { gen }\end{array}$ & M & GNAS Exons 1-12; heterozygot \\
\hline PHP-IC & $\begin{array}{l}\text { Multihormon- } \\
\text { resistenz }\end{array}$ & $\mathrm{AHO}$ & $\begin{array}{l}\text { Kognitive Einschränkun- } \\
\text { gen }\end{array}$ & M & GNAS Exon 13; heterozygot \\
\hline \multirow[t]{2}{*}{ PHP-Ib } & $\begin{array}{l}\text { PTH- und partielle } \\
\text { TSH-Resistenz }\end{array}$ & $\begin{array}{l}\text { Intrauterines Wachstum } \uparrow \\
\text { Brachydaktylie }\end{array}$ & $\begin{array}{l}\text { Methylierungsverlust } \\
\text { Exon A/B }\end{array}$ & M & $\begin{array}{l}\text { Imprintingdefekt: Deletionen STX16 oder } \\
\text { regulatorische Elemente des GNAS-Kom- } \\
\text { plexes }\end{array}$ \\
\hline & - & - & $\begin{array}{l}\text { Methylierungsdefekt - } \\
\text { multiple DMRs (spora- } \\
\text { disch) }\end{array}$ & - & $\begin{array}{l}\text { Paternale } 20 \text { q-Disomie od. unbekannter } \\
\text { epigenetischer Defekt (sporadisch) }\end{array}$ \\
\hline PPHP & Keine & $\begin{array}{l}\text { AHO; Intrauterines } \\
\text { Wachstum } \downarrow\end{array}$ & - & $\mathrm{P}$ & Heterozygot GNAS \\
\hline $\mathrm{POH}$ & Keine & $\begin{array}{l}\text { Progressive heterotope } \\
\text { Ossifikation }\end{array}$ & - & $\mathrm{P}$ & Heterozygot GNAS \\
\hline $\mathrm{OC}$ & None & $\begin{array}{l}\text { Heterotope Ossifikation - } \\
\text { Dermis und Subkutis }\end{array}$ & - & $P$ & Heterozygot GNAS \\
\hline
\end{tabular}

like Neuroendokrines sekretorisches Protein.

- $A / B$ wird ubiquitär, aber ausschließlich vom paternalen GNAS-Allel exprimiert.

\section{Genetische Diagnostik - Methodik und Vererbung}

\section{Pseudohypoparathyreoidismus des Typs 1a, $1 \mathrm{~b}$ und 1c, Pseudo- pseudohypoparathyreoidismus, Progressive Ossäre Heteroplasie und Osteoma cutis}

- werden durch inaktivierende GNASVarianten verursacht. Der Vererbungsmodus ist autosomal dominant (außer bei sporadischen 1bFällen - siehe - Tab. 2). Autosomal dominante Vererbung von Keimbahnmutationen bedeutet, dass für Nachkommen der Träger der pathogenen Variante ein 50-prozentiges Risiko besteht, ebenfalls Träger dieser Variante zu sein. Im Falle imprinteter Gene hängt der Phänotyp jedoch vom Geschlecht des Überträgers ab.

- $38 \%$ der erkrankten Patienten haben einen betroffenen Elternteil, $38 \%$ haben die pathogene GNAS-Variante de novo, und bei den restlichen $20-25 \%$ ist die Ursache unbekannt [9].
- Ist das väterliche GNAS-Allel von der inaktivierenden pathogenen Variante betroffen, so kommt es zur Albright'schen hereditären Osteodystrophie ohne Hormonresistenz (Pseudopseudohypoparathyreoidismus, PPHP) mit Kleinwuchs und verkürzten Metakarpalen IV und V.

- Liegt die inaktivierende Mutation auf dem mütterlich vererbten Allel, so ist die AHO mit Hormonresistenz (Pseudohypoparathyreoidismus Typ 1a) assoziiert. Neben der Knochenbeteiligung liegt eine PTH-Resistenz (Hypokalzämie) und manchmal auch eine Teil-Resistenz gegenüber TSH und den Gonadotropinen vor.

- Dem Pseudohypoparathyreoidismus Typ 1b liegen Methylierungsdefekte des GNAS-Lokus (assoziiert mit renaler PTH-Resistenz, aber oft ohne skelettale Auffälligkeiten) zugrunde. Aus genetischer Sicht sind zwei verschiedene Szenarien möglich - einerseits sind $A / B$ und STX16 (Syntaxin)-Gen bzw. regulatorische Bereiche von einem Methylierungsverlust betroffen, andererseits liegen Veränderungen in einer Reihe unterschiedlicher Methylierungsregionen (DMR differentially methylated regions) vor [10]. Eine seltene Variante des Typ $1 \mathrm{~b}$ ist die paternale uniparentale Disomie des Chromosoms 20, die bedeutet, dass anstatt eines maternalen und eines paternalen Allels zwei väterliche Allele vorliegen.

- Die häufigsten pathogenen GNASVarianten sind Frameshift-, Nonsense- oder Splicing-Mutationen, die zu keiner oder fehlerhafter Proteinbildung führen. Obwohl einzelne Hotspots (z. B. 4-bp-Deletion in Exon 7) beschrieben wurden, ist der Großteil der detektierten pathogenen Varianten über das ganze Gen verteilt. Für einige pathogene Varianten wurde auch eine Assoziation mit stärker ausgeprägter ektoper Ossifikation berichtet [11].

- Aufgrund der oben angeführten unterschiedlichen zugrundeliegenden molekulargenetischen Defekte ist die Entscheidung, welche Strategie zur Detektion der pathogenen Varianten gewählt wird, schwierig und verlangt ein profundes Kennen des komplexen genetischen Hintergrunds GNAS-assozierter Erkrankungen.

Optional können sowohl eine GNASEinzelgenanalyse (PCR-basierte Sequenzierung und MLPA), eine Methylierungsanalyse [12] oder ein MultigenPanel, insbesondere wenn eine Differen- 
tialdiagnose mit anderen Erkrankungen (z.B. NF1) von Relevanz ist, angedacht werden. Wie schon in vorhergehenden Beiträgen des Genetischen Alphabets angeführt, ist die Entscheidung für Multigen-Panels bzw. Exom- oder GenomSequencing auch mit einer durchaus herausfordernden humangenetischen Beratung vor und nach der genetischen Analyse verbunden und muss entsprechend vorbereitet sein.

\section{McCune-Albright-Syndrom (MAS)/ Fibröse Dysplasie (FD)}

- Während das MAS eine Prävalenz von 1-9/1.000.000 hat, wird geschätzt, dass die FD für $7 \%$ aller benignen Knochentumoren verantwortlich zeichnet. Beide Geschlechter sind gleich häufig von MAS/FD betroffen. In vielen PatientInnen erfolgt die Diagnose von Fibröser Dysplasie (FD) bzw. MAS phänotypisch, basierend auf dem Vorhandensein von zwei oder mehr typischen klinischen Symptomen.

- Bei unklarer oder schwach ausgeprägter Symptomatik ist ein Gentest hilfreich.

- Aktivierende pathogene Varianten, die im McCune-Albright-Syndrom resultieren, sind üblicherweise Missense-Mutationen, hauptsächlich in Exon 8 (p.(Arg201His); p.(Arg201Cys)) und seltener in Exon 9 (p.(Gln227Leu)). Sie betreffen zwei Aminosäurereste, die katalytisch für die GTPase-Aktivität relevant sind.

- Bei FD und MAS muss, wie schon oben erwähnt, zur Bestätigung der Diagnose nach aktivierenden somatischen GNAS-Varianten gesucht werden, wobei die Nachweisbarkeit auch von der Ausprägung des Mosaiks und der analytischen Sensitivität der Methodik abhängt. Die vielversprechendste Vorgangsweise ist, eine vom betroffenen Gewebe entnommene Probe auf die oben angeführten pathogenen Varianten zu testen - häufig verwendet werden PCRbasierte Sequenzierung oder TaqMan-Assays oder auch NGS (Next Generation Sequencing). Große Deletionen werden kaum beobachtet, andere pathogene Varianten als die oben beschriebenen werden selten detektiert und müssen häufig als Varianten unbekannter Signifikanz (VUS) klassifiziert werden.

- Da beim MAS keine GNAS-Keimbahnmutation vorliegt, besteht für Nachkommen und Geschwister der PatientInnen kein erhöhtes Risiko (mit der Normalbevölkerung verglichen), an MAS zu erkranken. Eine pränatale Diagnostik ist daher nicht angezeigt.

\section{Genetische Diagnostik - Veranlassung und Beratung}

Bevor eine humangenetische Analyse durch zuständige, einschlägige FachärztInnen veranlasst und im Labor durchgeführt wird, ist die Patientin/der Patient entsprechend aufzuklären und zu beraten. Diese Beratung muss dokumentiert werden, und die PatientIn/der Patient hat der Analyse schriftlich zuzustimmen. Das Ergebnis der genetischen Analyse muss in schriftlicher Form mitgeteilt und mit einer genetischen Beratung abgeschlossen werden. Die PatientInnen können die Durchführung der Analyse bzw. die Mitteilung des Ergebnisses zu jedem Zeitpunkt und ohne Angabe von Gründen widerrufen.

\section{Korrespondenzadresse}

Univ.-Prof. DI Dr. Sabina Baumgartner-

\section{Parzer}

Universitätsklinik für Innere Medizin III,

Medizinische Universität Wien

Währinger Gürtel 18-20, 1090 Wien, Österreich sabina.baumgartner-parzer@meduniwien.ac.at

Funding. Open access funding provided by Medical University of Vienna.

\section{Einhaltung ethischer Richtlinien}

Interessenkonflikt. A. Baumgartner und S. Baumgartner-Parzer geben an, dass kein Interessenkonflikt besteht.

Für diesen Beitrag wurden von den Autoren keine Studien an Menschen oder Tieren durchgeführt. Für die aufgeführten Studien gelten die jeweils dort angegebenen ethischen Richtlinien.

Open Access. Dieser Artikel wird unter der Creative Commons Namensnennung 4.0 International Lizenz veröffentlicht, welche die Nutzung, Vervielfältigung Bearbeitung, Verbreitung und Wiedergabe in jeglichem Medium und Format erlaubt, sofern Sie den/die ursprünglichen Autor(en) und die Quelle ordnungsgemäß nennen, einen Link zur Creative Commons Lizenz beifügen und angeben, ob Änderungen vorgenommen wurden.

Die in diesem Artikel enthaltenen Bilder und sonstiges Drittmaterial unterliegen ebenfalls der genannten Creative Commons Lizenz, sofern sich aus der Abbildungslegende nichts anderes ergibt. Sofern das betreffende Material nicht unter der genannten Creative Commons Lizenz steht und die betreffende Handlung nicht nach gesetzlichen Vorschriften erlaubt ist, ist für die oben aufgeführten Weiterverwendungen des Materials die Einwilligung des jeweiligen Rechteinhabers einzuholen.

Weitere Details zur Lizenz entnehmen Sie bitte der Lizenzinformation auf http://creativecommons.org/ licenses/by/4.0/deed.de.

\section{Literatur}

1. Haldeman-EnglertCR, Hurst ACE, Levine MA (2017) Disorders of GNAS inactivation. In: Adam MP, Ardinger HH, Pagon RA et al (Hrsg) GeneReviews ${ }^{\circledR}$ (https://pubmed.ncbi.nlm.nih.gov/29072892/)

2. OMIM (2018) 139320 GNAS complex locus. https:// www.omim.org/entry/139320. Zugegriffen: 20. Mai 2020

3. Javaid MK, Boyce A, Appelman-Dijkstra N, Ong J, Defabianis P, Offiah A, Arundel P, Shaw N, Dal Pos V, Underhil A, Portero D, Heral L, Heegaard A-M, Masi L, Monsell F, Stanton R, Dijkstra PDS, Brandi ML, Chapurlat R, Hamdy NAT, Collins MT (2019) Best practice management guidelines for fibrous dysplasia/McCune-Albright syndrome: a consensus statement from the FD/MASinternational consortium. Orphanet J Rare Dis 14(1):139. https://doi.org/10.1186/s13023019-1102-9

4. Campbell CD, EichlerEE (2013) Properties and rates of germline mutations in humans. Trends Genet 29:575-584. https://doi.org/10.1016/j.tig.2013. 04.005

5. Aldred MA, Trembath RC (2000) Activating and inactivating mutations in the human GNAS1 gene. Hum Mutat 16(3):183-189. https://doi. org/10.1002/1098-1004(200009)16:3<183::AIDHUMU1>3.0.CO;2-L

6. Linglart A, Carel JC, Garabédian M, Lé T, Mallet $E_{\text {, }}$ Kottler ML (2002) GNAS1 lesions in pseudohypoparathyroidism la and Ic: genotype phenotype relationship and evidence of the maternal transmission of the hormonal resistance. J Clin Endocrinol Metab 87(1):189-197. https://doi.org/ 10.1210/jcem.87.1.8133

7. Shore EM, Ahn J, de Beur JS, Li M, Xu M, Gardner RJ, Zasloff MA, Whyte MP, Levine MA, Kaplan FS (2002) Paternally inherited inactivating mutations of the GNAS1 gene in progressive osseous heteroplasia. N Engl J Med 346(2):99-106. https://doi.org/10. 1056/NEJMoa011262

8. Levine MA (2012) An update on the clinical and molecular characteristics of pseudohypoparathyroidism. Curr Opin Endocrinol Diabetes Obes 19(6):443-451. https://doi.org/10.1097/ MED.0b013e32835a255c

9. Elli FM, Linglart A, Garin I, de Sanctis L, Bordogna P, Grybek V, Pereda A, Giachero F, Verrua E, Hanna P, Mantovani G, Perez de Nanclares G (2016) The 
prevalence of GNAS deficiency-related diseases in a large cohort of patients characterized by the EuroPHP network. J Clin Endocrinol Metab 101(10):3657-3668. https://doi.org/10.1210/jc 2015-4310

10. Linglart $A$, Gensure RC, Olney $R C$, Jüppner $H$, Bastepe M (2005) A novel STX16 deletion in autosomal dominant pseudohypoparathyroidism type $\mathrm{lb}$ redefines the boundaries of a cis-acting imprinting control element of GNAS. Am J Hum Genet 76(5):804-814. https://doi.org/10.1086/ 429932

11. Lemos MC, Thakker RV (2015) GNAS mutations in pseudohypoparathyroidism type $1 \mathrm{a}$ and related disorders. Hum Mutat 36:11-19. https://doi.org/ 10.1002/humu.22696

12. Elli FM, de Sanctis L, Bollati V, Tarantini L, Filopant M, Barbieri AM, Peverelli E, Beck-Peccoz P, Spada A, Mantovani G (2014) Quantitative analysis of methylation defects and correlation with clinical characteristics in patients with pseudohypoparathyroidism type I and GNAS epigenetic alterations. J Clin Endocrinol Metab 99(3):E508-E517. https:// doi.org/10.1210/jc.2013-3086

Hinweis des Verlags. Der Verlag bleibt in Hinblick auf geografische Zuordnungen und Gebietsbezeichnungen in veröffentlichten Karten und Institutsadressen neutral.

\section{Eine gesunde Lebensweise senkt das Risiko für Diabetes und Folgeerkrankungen}

Wissenschaftlerinnen und Wissenschaftler haben untersucht, inwiefern Menschen mit einer gesunden Lebensweise ihr Risiko, an Typ-2-Diabetes zu erkranken, mindern - und ob sich ein insgesamt gesunder Lebensstil positiv auf Diabetes-Folgeerkrankungen und das Sterberisiko auswirkt.

Beim Lebensstil gilt das Prinzip: „Viel Gesundes hilft viel“. Das heißt: Wer eine umfassende gesunde Lebensweise konsequent durchhält, also mehrere Aspekte eines gesunden Lebensstils kombiniert - regelmäßige Bewegung, gesunde Ernährung, Gewichtskontrolle, Rauchstopp, ausreichend Schlaf und moderater Alkoholgenuss - kann sowoh sein Risiko, an Typ-2-Diabetes zu erkranken, als auch das Risiko für Diabetes-Folgeerkrankungen und einen frühzeitigen Tod deutlich senken. Das zeigt eine systematische Studienübersicht zu diesen Zusammenhängen. Chinesische Wissenschaftlerinnen und Wissenschaftler haben dabei 2 unterschiedliche Auswirkungen eines gesunden Lebensstils eingehend untersucht: 1. das Risiko, an Typ2-Diabetes zu erkranken, und 2. die gesundheitlichen Folgen für Menschen mit Typ-2Diabetes. Für die erste Analyse wurden Daten von etwa 1 Million Teilnehmenden aus insgesamt 14 Studien zusammengefasst, wohingegen die zweite Analyse auf etwa 34.000 Personen mit Typ-2-Diabetes aus 10 Studien beruhte.

\section{Diabetes-Risiko fällt deutlich}

Die Auswertung zum Zusammenhang von einer gesunden Lebensweise (mindestens 3 Faktoren, beispielsweise gesunde Ernährung, Normalgewicht und regelmäßige körperliche Aktivität) und dem Diabetes-Risiko hat gezeigt, dass diejenigen, die am gesündesten lebten, ihr Risiko für Typ-2-Diabetes im Vergleich zur Gruppe, die am ungesündesten lebte, um 75 Prozent senken konnte. Das galt für Personen mit unterschiedlichsten Voraussetzungen, was etwa das Alter, den sozialen Status oder das Herkunftsland betrifft.

\section{Gesunder Lebensstil schützt auch vor Herz-Kreislauf-Erkrankungen}

In den Studien zum Zusammenhang von einem gesunden Lebensstil und dem Sterberisiko sowie dem Risiko für Herz-KreislaufErkrankungen bei Patientinnen und Patienten mit Typ-2-Diabetes ergaben sich ebenfalls deutliche Vorteile eines umfassenden gesunden Lebensstils. Mit der gesündesten Lebensweise ließ sich das allgemeine Sterberisiko um 56 Prozent und das Risiko, infolge einer Herz-Kreislauf-Erkrankung zu versterben, um 49 Prozent senken. Das Risiko, eine Herz-Kreislauf-Erkrankung zu entwickeln, war bei den Menschen mit Typ-2-Diabetes, die am gesündesten lebten, um 52 Prozent geringer als bei denen, die insgesamt sehr ungesund lebten.

\section{Gesünder leben lohnt sich}

Um gegen Diabetes sowie dessen Folgeerkrankungen, insbesondere Herz-KreislaufErkrankungen, vorzubeugen, sei es also wichtig, die Menschen für eine umfassende gesunde Lebensweise zu motivieren, schlussfolgert das Forschungsteam.

\section{Orignalpublikation:}

Zhang, Y. et al.: Combined lifestyle factors and risk of incident type 2 diabetes and prognosis among individuals with type 2 diabetes: a systematic review and meta-analysis of prospective cohort studies. In: Diabetologia, 2020, 63: 21-33

Quelle: diabinfo

(Das Diabetesinforamtionsportal) am Helmholtz Zentrum München, Deutschen Diabetes-Zentrum und Deutschen Zentrum für Diabetesforschung, www.diabinfo.de 